\title{
BMJ Open Developing and validating a risk prediction model for acute care based on frailty syndromes
}

\author{
J Soong,,${ }^{1,2}$ A J Poots, ${ }^{1}$ S Scott, ${ }^{3} \mathrm{~K}$ Donald, ${ }^{3} \mathrm{D}$ Bell ${ }^{1}$
}

To cite: Soong J, Poots AJ, Scott S, et al. Developing and validating a risk prediction model for acute care based on frailty syndromes. BMJ Open 2015;5:e008457. doi:10.1136/bmjopen-2015008457

- Prepublication history and additional material is available. To view please visit the journal (http://dx.doi.org/ 10.1136/bmjopen-2015008457).

Received 9 April 2015 Revised 30 July 2015 Accepted 19 August 2015

\section{SLinked}

- http://dx.doi.org/10.1136/ bmjopen-2015-008456

CrossMark

\begin{abstract}
${ }^{1} \mathrm{NIHR}$ CLAHRC Northwest London, Imperial College London, Chelsea and Westminster Campus, London, UK

${ }^{2}$ Royal College of Physicians, London, UK

${ }^{3}$ Oliver Wyman, London, UK
\end{abstract}

Correspondence to

Dr J Soong;

johnsoong@imperial.ac.uk

\section{ABSTRACT}

Objectives: Population ageing may result in increased comorbidity, functional dependence and poor quality of life. Mechanisms and pathophysiology underlying frailty have not been fully elucidated, thus absolute consensus on an operational definition for frailty is lacking. Frailty scores in the acute medical care setting have poor predictive power for clinically relevant outcomes. We explore the utility of frailty syndromes (as recommended by national guidelines) as a risk prediction model for the elderly in the acute care setting.

Setting: English Secondary Care emergency admissions to National Health Service (NHS) acute providers.

Participants: There were $\mathrm{N}=2099252$ patients over 65 years with emergency admission to NHS acute providers from 01/01/2012 to $31 / 12 / 2012$ included in the analysis.

Primary and secondary outcome measures: Outcomes investigated include inpatient mortality, 30-day emergency readmission and institutionalisation. We used pseudorandom numbers to split patients into train (60\%) and test $(40 \%)$. Receiver operator characteristic (ROC) curves and ordering the patients by deciles of predicted risk was used to assess model performance. Using English Hospital Episode Statistics (HES) data, we built multivariable logistic regression models with independent variables based on frailty syndromes (10th revision International Statistical Classification of Diseases, Injuries and Causes of Death (ICD-10) coding), demographics and previous hospital utilisation. Patients included were those $>65$ years with emergency admission to acute provider in England (2012).

Results: Frailty syndrome models exhibited ROC scores of 0.624-0.659 for inpatient mortality, 0.630.654 for institutionalisation and $0.57-0.63$ for 30 -day emergency readmission.

Conclusions: Frailty syndromes are a valid predictor of outcomes relevant to acute care. The models predictive power is in keeping with other scores in the literature, but is a simple, clinically relevant and potentially more acceptable measurement for use in the acute care setting. Predictive powers of the score are not sufficient for clinical use.

\section{Strengths and limitations of this study}

- It is a simple clinical model that has moderate predictive powers outcomes relevant to acute medical care. It has reduced data requirements compared with existing frailty models trialled in the acute care setting with predictive powers evenly spread over three outcomes.

- It is a model designed to be that could be applied at point of access to acute care, does not rely on self-reported data and was derived from whole population data that are routinely collected.

- This study adds to emerging knowledge surrounding the secondary use of administrative data. It provides a novel methodology to best utilise routinely collected data in a systematic and robust manner that minimises limitations and optimises data quality and reliability.

- Hospital Episode Statistics (HES) is retrospectively coded, thus reflects the patient's condition at discharge from hospital.

- Diagnostic coding accuracy in HES has been challenged.

\section{INTRODUCTION}

In the majority of countries, the population is living to a greater age. This change in population demographics is not necessarily associated with failing health as individual variation exists. A recent survey indicates that the majority of those over 80 years are satisfied or very satisfied with their health. ${ }^{1}$ For some, however, this is associated with an increase in comorbidity ${ }^{2}$ and functional dependence, ${ }^{3}$ with a consequent higher health and social care cost. A large component of this increased need is reflected in hospital demand both for elective and non-elective care. Patients over the age of 65 constitute two-thirds of admissions, $40 \%$ of all hospital bed days and $65 \%$ of National Health Service (NHS) spend in acute care. ${ }^{4}$ Within this population, there is group of patients that 
most clinicians and the public would regard or recognise as frail and at higher risk of adverse outcomes.

Much research has taken place in understanding the pathophysiology and mechanisms underlying frailty; ${ }^{5} 6$ however, assessing frailty reliably remains problematic and is a research priority. ${ }^{7-13}$ This is compounded at present by the absence of consensus on an operational definition of frailty. ${ }^{14-16}$ Two broad approaches to measuring frailty have been described; a specific biophysical phenotype (unintentional weight loss, exhaustion, weakness, slowness and low physical activity) ${ }^{17}$ and an index of accumulated deficit model. ${ }^{18}$ These models have the benefit of reproducibility, and predict important health outcomes such as mortality, self-reported health and functional dependency. ${ }^{19}$ Though overlap exists between these models, ${ }^{20}$ to date, published scores based on these operational definitions demonstrate only poor to moderate predictive powers for adverse outcomes within the acute medical care setting. ${ }^{9}$ Developing a reliable and clinically acceptable method to quantify frailty that links to outcomes would help in clinical practice as well as provide a method for longitudinal population analysis.

Within elderly care, there are a number of syndromes that are commonly recognised in older person, including 'Giants of geriatrics' ${ }^{21}$ or geriatric syndromes. ${ }^{5}$ These are common clinical presentations of multifactorial illdefined processes recognised in older persons. They include cognitive impairment, pressure ulcers, mobility problems, falls and incontinence. Conceptually, they represent a final common pathway of concentric, non-linear processes formed by the interaction between aetiological and physiological mechanisms, as yet not fully elucidated. ${ }^{5}$ When complex systems fail, high-order systems tend to break down first. ${ }^{22}$ This potentially makes frailty syndromes a robust marker for this vulnerable patient cohort. In the acute care setting, they are associated with increased functional dependence and length of hospital stay. ${ }^{23}$ Current national guidelines for the care of the older person in acute care recommend using frailty syndromes as a possible methodology to assess for frailty. ${ }^{11} 12$

This study explores the hypothesis that frailty syndromes are a valid measure of adverse health outcomes in older persons within the acute care population in England using routinely available secondary care data based on Hospital Episode Statistics (HES) ${ }^{24}$ We aim to develop and validate a model of frailty based on these syndromes as the first steps of developing a sensitive clinically relevant assessment tool to be used at point of access of acute care. We aim to evaluate its predictive power for clinical outcomes relevant to acute medical care. For construct validity, ${ }^{25}$ we explore its association with the Charlson comorbidity score. ${ }^{26}$

\section{METHODS}

\section{Data source}

HES is an administrative data set collected for the secondary care setting that has high levels of data completeness and rigorous data cleaning processes, ensuring high data quality. Each record in HES corresponds to a finished consultant episode, during which a patient is under the care of an individual consultant. These episodes were aggregated into hospital spells covering the entirety of a patient's length of stay in a hospital using established methodology. ${ }^{27}$

HES contains 20 fields per record for diagnoses codes that are defined in the 10th revision of the International Statistical Classification of Diseases, Injuries and Causes of Death (ICD-10). We systematically explored all 20 diagnostic fields within HES for ICD-10 diagnostic codes to group together to form frailty syndromes (see online supplementary appendix 1). To explore the effect of coding shifts over time within HES (thereby potentially affecting coding reliability), annual trend profiles for the grouped ICD-10 diagnostic codes were plotted from January 2005 to March 2013 (see online supplementary appendix 2). As a result of this analysis, data from the years 2010-2012 were selected for the final model, and we merged ICD-10 diagnostic codes for dementia, delirium and senility to form a unified frailty syndrome (cognitive impairment).

Emergency admissions were defined as those for which the method of admission was recorded as 'Emergency', either via accident and emergency services, a general practitioner, a Bed Bureau, a consultant outpatient clinic or other means (HES Column header: admimeth=21, 22, 23, 24, 28).

The final risk prediction model included all spells for patients over 65 years with emergency admission to English NHS acute providers from 01/01/2012 to 31/ 12/2012 ( $\mathrm{N}=2099$ 252).

\section{Model input and output variables}

Table 1 describes predictor variables for study, including patient demographics, frailty syndromes and previous service use. Table 2 describes outcome variables under investigation, including inpatient mortality, 30-day emergency readmission and increase functional dependence at discharge (measured as a change in discharge destination to an institution providing more social and functional support when compared with admission source). In the UK, residential homes are care homes that provide accommodation, meals and some personal care. Nursing homes are residential care homes, but additionally have registered nurses that provide care for more complex needs. English care homes can be privately owned, third sector, local authority or NHS owned. In England, cost for local authority part 3 residential accommodation is charged to the resident.

The model consisted of both historical and withinspell variables. Historical variables included data up to 24 months prior to admission spell in 2012, while withinspell variables were only measured during the patients' admission spell in 2012. Historical diagnostic codes were chosen over in-spell ones when coding for frailty syndromes as this more accurately described a risk 
Table 1 Predictor inputs for frailty risk prediction model (independent variables)

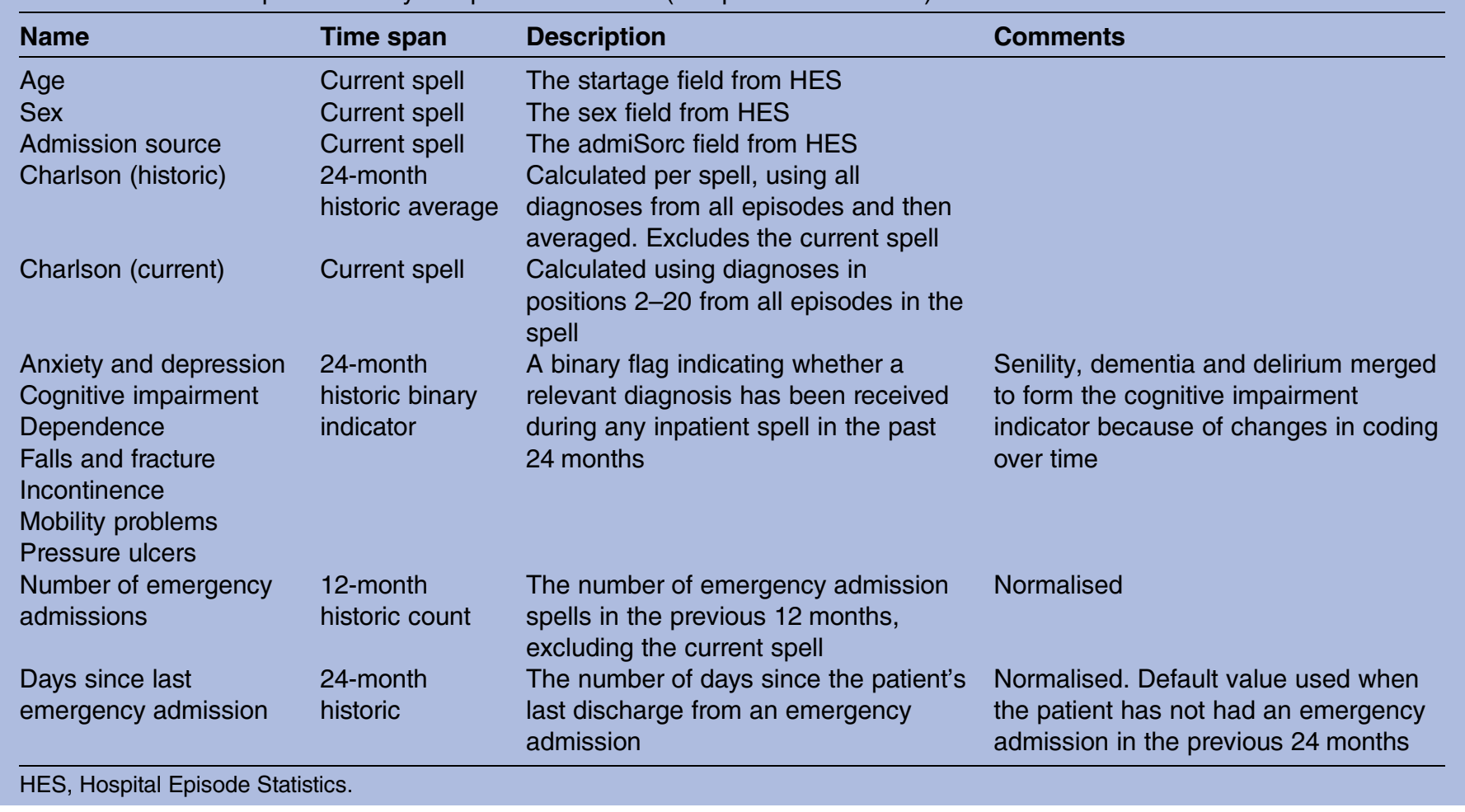

prediction model at the point of access to acute care. Charlson comorbidity scores were calculated in HES using previously described methodology, ${ }^{28}$ using weightings originally described by Charlson. ${ }^{26}$

Spells ending with inpatient mortality were excluded when predicting institutionalisation or readmission within 30 days. Spells where the admission source or discharge destination could not be allocated a tier were also excluded when calculating functional dependence (approximately $<1 \%$ of spells not ending in mortality).

\section{Model development and testing}

Pseudorandom numbers split patients into train $(60 \%)$ and test $(40 \%)$ groups. We then split spells into train (1 259185 spells) and test (840 067 spells) sets based on the groupings (to ensure no patient appears in both

Table 2 Predictor outputs of frailty risk prediction model (dependant variables)

\begin{tabular}{|c|c|c|c|c|}
\hline \multicolumn{2}{|c|}{ Name } & Time span & Description & Comments \\
\hline \multirow{3}{*}{\multicolumn{2}{|c|}{$\begin{array}{l}\text { Inpatient mortality } \\
\text { 30-day emergency } \\
\text { readmission } \\
\text { Increase in functional } \\
\text { dependence }\end{array}$}} & Current spell & Indicates if the discharge destination was death & \\
\hline & & $\begin{array}{l}30 \text { days from } \\
\text { discharge }\end{array}$ & $\begin{array}{l}\text { Indicates if the patient had an emergency admission within } \\
30 \text { days of discharge from the current spell }\end{array}$ & \\
\hline & & Current spell & $\begin{array}{l}\text { Binary outcome indicates if the patient's discharge destination } \\
\text { was associated with a higher level of functional dependence }\end{array}$ & $\begin{array}{l}\text { See functional } \\
\text { dependence tiers }\end{array}$ \\
\hline Tier & \multicolumn{4}{|c|}{ Values in tier } \\
\hline \multirow[t]{2}{*}{1} & \multirow{2}{*}{\multicolumn{4}{|c|}{$\begin{array}{l}\text { The usual place of residence, including no fixed abode } \\
\text { Temporary place of residence when usually resident elsewhere, for examp } \\
\text { establishments }\end{array}$}} \\
\hline & & & & \\
\hline \multirow[t]{2}{*}{2} & \multirow{2}{*}{\multicolumn{4}{|c|}{$\begin{array}{l}\text { Local authority part } 3 \text { residential accommodation: where care is provided } \\
\text { Non-NHS (other than Local Authority) run residential care home }\end{array}$}} \\
\hline & & & & \\
\hline \multirow[t]{2}{*}{3} & \multicolumn{4}{|c|}{ NHS run nursing home, residential care home or group home } \\
\hline & \multicolumn{4}{|c|}{ Non-NHS (other than Local Authority) run nursing home } \\
\hline 4 & \multicolumn{4}{|c|}{$\begin{array}{l}\text { NHS other hospital provider: ward for general patients or the younger physically disabled or A\&E department } \\
\text { Non-NHS run hospital }\end{array}$} \\
\hline 5 & \multicolumn{4}{|c|}{ Non-NHS (other than Local Authority) run hospice } \\
\hline
\end{tabular}


train and test sets). Multicollinearity between predictor variables was investigated by variance inflation factor (VIF), where VIF scores of over 3 were taken to denote unacceptable collinearity. Scikit-learn ${ }^{29}$ implementation of logistic regression with 12 regularisation was used to create the risk prediction model. The model coefficients selected in the train set were then used to score all samples in the test set. Finally, receiver operator characteristic (ROC) curves and area under the curve (AUC) scores ${ }^{30}$ were generated based on the predicted probabilities within the test set scores. Hosmer-Lemeshow ${ }^{31}$ tests with scipy implementation of Pearson's $\chi^{2}$ test were performed for goodness-of-fit. Ordering the patients by deciles of predicted risk allows a visual representation of the models discrimination.

\section{RESULTS}

\section{Mortality}

None of the models predictor variables (patient demographics, frailty syndromes, previous service use) demonstrated unacceptable collinearity (1.1-2.8) (table 3). Table 4 describes the predictive power of various frailty syndrome models for within-spell inpatient mortality (range of AUCs 0.624-0.659). The frailty syndromes and admission history model demonstrates moderate discriminatory power, with the top $10 \%$ of patients identified at highest risk of inpatient mortality having a mortality rate $(13 \%)$ nearly twice the average population (7\%; figure 1). The addition of Charlson comorbidity score did not significantly improve the predictive power of the model ( $\mathrm{AUC}=0.641$ ). However, in-spell Charlson and frailty syndrome models described slightly improved predictive power over historical models (tables 4 and 5).

\section{Discharge to a higher level of support}

Table 6 describes the predictive power of frailty syndrome models to predict discharge to a higher level of support (institutionalisation; range of AUCs 0.63-0.654). The frailty syndromes and admission source model demonstrated moderate discriminatory power, with the top $10 \%$ of patients identified at highest risk of being discharged to a higher level of support (17\%) at nearly

Table 3 Variance inflation factor scores for predictor variables

\begin{tabular}{ll}
\hline Variance inflation factor scores & \\
\hline Age & 2.6 \\
Sex & 1.8 \\
Historic Charlson & 1.1 \\
Anxiety and depression & 1.7 \\
Cognitive impairment & 1.1 \\
Dependence & 1.6 \\
Fall & 1.1 \\
Incontinence & 1.2 \\
Mobility & 1.1 \\
Pressure ulcers & 1.8 \\
\hline
\end{tabular}

twice the average population (9\%; figure 2). Historic Charlson comorbidity scores (taking into account age and gender) exhibited AUCs of 0.617.

\section{Thirty-day emergency readmission}

Table 7 describes the predictive power of the frailty models to predict emergency readmission within 30 days (range of AUCs 0.57-0.63). The frailty syndromes and admission history model demonstrated moderate discriminatory power, with the top $10 \%$ of patients identified at highest risk of emergency readmission within 30 days $(39 \%)$ at nearly twice the average population (21\%; figure 3). Historic Charlson comorbidity scores (taking into account age and gender) exhibited AUCs of 0.591 .

\section{DISCUSSION}

Risk stratification of older persons who require acute care is complex and challenging. Reliable recognition of frailty is a research and clinical priority for acute hospital care $^{7-13}$ to help inform routine clinical decisionmaking and plan appropriate care. To date, there is no routinely available and reliable clinical score for use within the acute care setting. This study explores the use of internationally recognised frailty syndromes coded within HES data to potentially aid more reliable frailty recognition within the hospital setting. HES data can reliably provide data related to mortality and high resource need (eg, occupied bed days or readmission). We have constructed a surrogate marker of functional dependency (ie, institutionalisation) using available HES fields. The ideal frailty assessment for acute care needs to be comprehensively multidimensional to avoid missing aspects of patient care that may contribute to further decline or harm. It needs to predict outcomes that are relevant to the patient, carers and to acute care providers. To be fit for purpose, it should be optimised for clinical usability: that is, simple, reliable, does not fully rely on self-reported or carer-reported data and possess high sensitivity if functioning as a screening tool. Ideally, there should be the ability to personalise the assessment and 'threshold' set to patient preference and previous level of functioning. It should be able to provide a method to measure frailty over the course of an episode of acute illness and over a patient's life as opposed to single isolated static measures. Ultimately, it should be able to highlight areas for intervention to prevent, reverse or minimise further decline.

Studies exploring the predictive power of frailty scores for outcomes relevant to the UK acute medical care setting (table 8 ) include prospective observational cohort studies ${ }^{8932}$ and secondary analysis of routinely collected large data sets, both clinical ${ }^{33}$ and administrative. ${ }^{28}{ }^{34}$ Our model performs uniformly across the clinical outcomes and is comparable in predictive power to frailty scores in the same setting. None of the models have predictive powers suitable for clinical risk prediction at the 
Table 4 Frailty syndrome models to predict within spell in-patient mortality

\begin{tabular}{|c|c|c|c|}
\hline Model & ORs & & AUC \\
\hline \multirow[t]{9}{*}{ Historical frailty syndromes model } & Age & 1.05 & 0.624 \\
\hline & Sex & 1.30 & \\
\hline & Anxiety and depression & 0.94 & \\
\hline & Cognitive impairment & 1.21 & \\
\hline & Functional dependence & 1.11 & \\
\hline & Falls and fracture & 0.94 & \\
\hline & Incontinence & 1.06 & \\
\hline & Mobility problems & 1.08 & \\
\hline & Pressure ulcers & 1.29 & \\
\hline \multirow[t]{9}{*}{ In-spell frailty syndromes model } & Age & 1.05 & 0.659 \\
\hline & Sex & 1.20 & \\
\hline & Anxiety and depression & 0.93 & \\
\hline & Cognitive impairment & 1.40 & \\
\hline & Functional dependence & 0.64 & \\
\hline & Falls and fracture & 0.65 & \\
\hline & Incontinence & 1.34 & \\
\hline & Mobility problems & 1.16 & \\
\hline & Pressure ulcers & 4.04 & \\
\hline \multirow[t]{10}{*}{ Historical frailty syndromes and Charlson comorbidity scores } & Age & 1.05 & 0.641 \\
\hline & Sex & 1.09 & \\
\hline & Charlson & 1.20 & \\
\hline & Anxiety and depression & 0.98 & \\
\hline & Cognitive impairment & 1.01 & \\
\hline & Functional dependence & 1.02 & \\
\hline & Falls and fracture & 0.97 & \\
\hline & Incontinence & 1.01 & \\
\hline & Mobility problems & 1.01 & \\
\hline & Pressure ulcers & 1.05 & \\
\hline \multirow[t]{11}{*}{ Historical frailty syndromes and admission history (final model) } & Age & 1.05 & 0.632 \\
\hline & Sex & 1.21 & \\
\hline & Anxiety and depression & 0.95 & \\
\hline & Cognitive impairment & 1.05 & \\
\hline & Functional dependence & 1.04 & \\
\hline & Falls and fracture & 0.90 & \\
\hline & Incontinence & 1.02 & \\
\hline & Mobility problems & 1.02 & \\
\hline & Pressure ulcers & 1.11 & \\
\hline & Number of emergencyadmissions (12 months) & 0.97 & \\
\hline & Days since last emergency admission & 0.79 & \\
\hline
\end{tabular}

patient's bedside (AUC >0.80). The exception to this is a single study in the Acute Medical Unit (AMU) setting in rural Ireland, ${ }^{35}$ which reported AUCs of $>0.8$ for 30-day mortality and functional decline, but the results of this secondary analysis of a clinical database was not reproduced in prospective observational study at a large teaching centre in the UK. ${ }^{10}$

Our model has notable strengths. It is a simple clinical model that has moderate predictive power outcomes relevant to acute medical care. It has less data requirements compared with the Frailty Index (36 input variables), ${ }^{9}$ Patient At Risk of Readmission 30-Day (PARR30; up to 18 input variables), ${ }^{34}$ Risk Index for Geriatric Acute Medical Admissions (RIGAMAs; 30 input variables) ${ }^{33}$ and Charlson comorbidity score (17 input variables). ${ }^{28}$ Importantly, in comparison to other scores, its predictive power appears to be evenly spread over the three outcomes and does not rely on self-reported data (eg, Identifying Seniors at Risk (ISAR) score) ${ }^{36}$ It is a model designed to be that could be applied at point of access to acute care. It was derived from whole population data that is routinely collected, with applicability at population and patient level. This study adds to emerging knowledge surrounding the secondary use of administrative data. It provides a novel methodology to best utilise routinely collected data in a systematic and robust manner that minimises limitations and optimises data quality and reliability.

Existing frailty scores in the acute care setting have very different input variables (thus likely do not measure the same thing). Optimal outcome variable selection is also yet unclear. For example, our model and most 


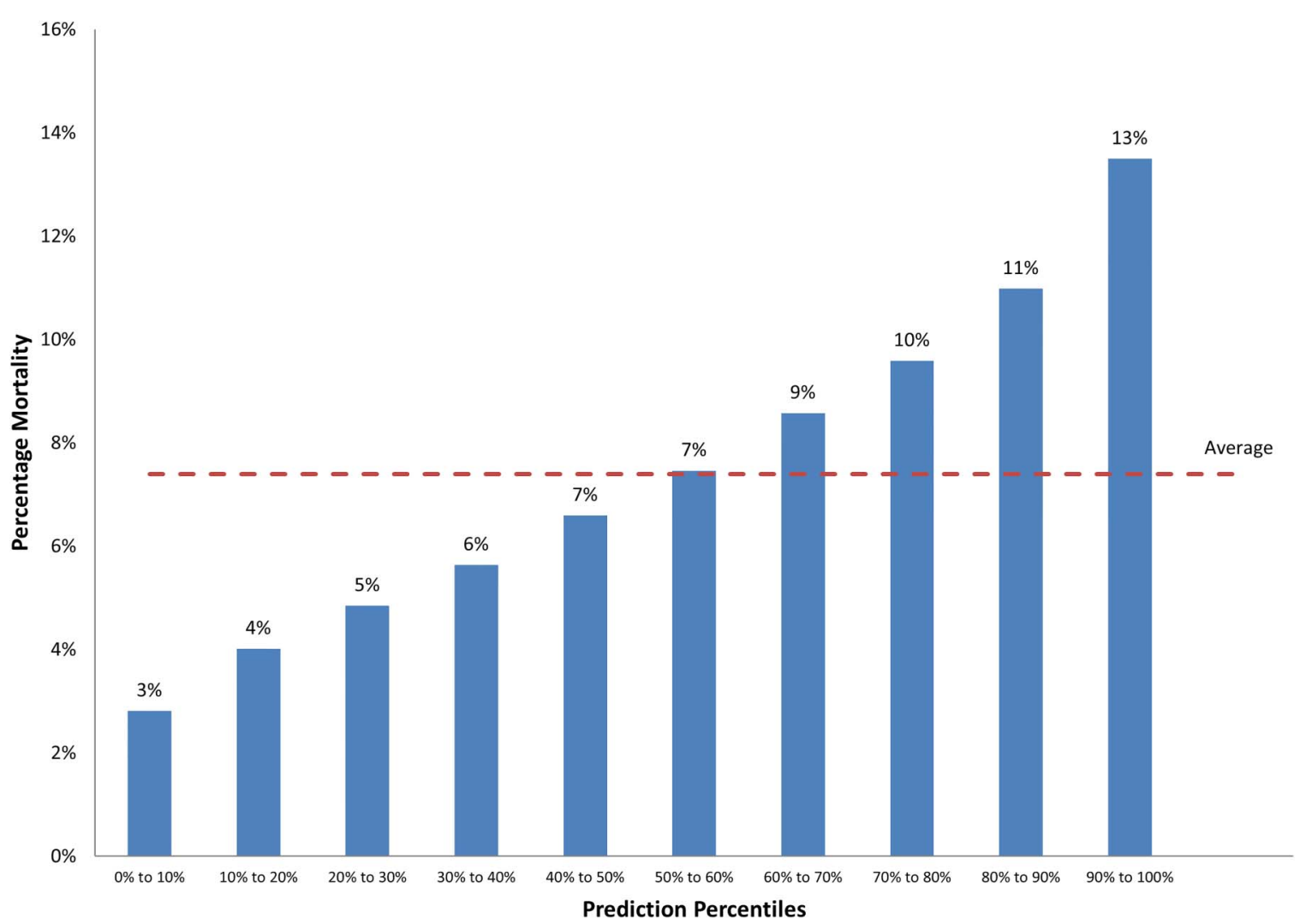

Figure 1 Percentage mortality by prediction ranking for the frailty syndromes and admission history model.

existing frailty scores do not take into account illness severity or disease acuity. We postulate that the addition of variables included in the National Early Warning Score (NEWS) ${ }^{37}$ may improve discrimination of frailty models. RIGAMAs ${ }^{33}$ notable predictive powers for inpatient mortality may reflect discrimination for acute critical illness given input variables that largely record physiological and metabolic derangement, including prognostic biomarkers (eg, troponin). However, it may be that the optimal outcome variable for frailty in acute care is 30-day or 90-day mortality.

Studies of frailty scores in the emergency department (ED) setting display similar predictive powers for a wide range of outcomes: HK-ISAR $>65$ years discharged from ED AUC 0.59-0.62 for composite outcome of institutionalisation, reattendance or death ${ }^{38}$; ISAR score $>65$ years

Table 5 Charlson comorbidity models to predict within spell in-patient mortality

\begin{tabular}{llll}
\hline Model & ORs & & AUC \\
\hline Historic Charlson & Age & 1.05 & 0.639 \\
& Sex & 1.31 & \\
\multirow{4}{*}{ In-spell Charlson } & Charlson & 1.20 & \\
& Age & 1.05 & 0.681 \\
& Sex & 1.02 & \\
& Charlson & 1.29 & \\
\hline AUC, area under the curve.
\end{tabular}

admitted to hospital via ED AUC $0.549-0.584,{ }^{39}$ AUC 0.66 for depressive symptoms, AUC $0.61-0.68$ for frequent ED visits, AUC 0.66-0.68 for frequent hospitalisation, AUC of 0.71 for frequent use of community services, ${ }^{40}$ high acute care utilisation AUC $0.68 ;{ }^{41}$ Triage Risk Screening Tool (TRST) score AUC 0.626-0.640 and Variable Indicative of Placement risk (VIP) score AUC $0.588-0.654$ for functional decline $>65$ years admitted to hospital via ED; ${ }^{39}$ Score Hospitalier d'Evaluation du Risque de Perte d'Autonomie (SHERPA) for $>70$ years admitted via ED AUC 0.73 for functional decline at 3 months; ${ }^{42}$ Hospital Admission Risk Profile (HARP) $>70$ years admitted to hospital AUC 0.65 for functional decline ${ }^{43}$

Studies of frailty scores in the hospital ward setting report slightly better predictive powers, but these scores might reflect a subselected (and therefore possibly more frail), and in most instances, older patient population: $>70$ years admitted to geriatric unit by clinical judgement for composite outcome of mortality OR admission to residential care facility OR transfer from low to high care within residential facility at discharge frailty index of accumulated deficits (FI-CD) AUC 0.735, Katz AUC 0.704, Cardiovascular Health Study (CHS) AUC 0.675, Study of Osteoporotic Fractures (SOF) AUC 0.679, Fatigue, Resistance Ambulation, Illness, Loss of Weight (FRAIL) AUC 0.638, ten-domain frailty index based on Comprehensive Geriatric Assessment (FI-CGA-10) AUC 0.617, Gait AUC 0.643, SHERPA AUC 0.697, Multi- 
Table 6 Frailty syndrome models to predict discharge with a higher level of support (institutionalisation)

\begin{tabular}{|c|c|c|c|}
\hline Model & ORs & & AUC \\
\hline \multirow[t]{11}{*}{ Historic frailty syndromes and admission history } & Age & 1.04 & \multirow[t]{11}{*}{0.634} \\
\hline & Sex & 0.94 & \\
\hline & Anxiety and depression & 0.98 & \\
\hline & Cognitive impairment & 1.36 & \\
\hline & Functional dependence & 1.20 & \\
\hline & Falls and fracture & 1.15 & \\
\hline & Incontinence & 1.09 & \\
\hline & Mobility problems & 1.12 & \\
\hline & Pressure ulcers & 1.20 & \\
\hline & Number of emergency admissions (past 12 months) & 0.82 & \\
\hline & Days since last emergency admission & 0.98 & \\
\hline \multirow[t]{10}{*}{ Historic frailty syndromes and admission source } & Age & 1.04 & \multirow[t]{10}{*}{0.654} \\
\hline & Sex & 0.94 & \\
\hline & Admission source $(\times 5)$ & $0.42-2.60$ & \\
\hline & Anxiety and depression & 0.94 & \\
\hline & Cognitive impairment & 1.36 & \\
\hline & Functional dependence & 1.17 & \\
\hline & Falls and fracture & 1.14 & \\
\hline & Incontinence & 1.08 & \\
\hline & Mobility problems & 1.16 & \\
\hline & Pressure ulcers & 1.17 & \\
\hline \multirow[t]{9}{*}{ Historic frailty syndromes } & Age & 1.05 & \multirow[t]{9}{*}{0.63} \\
\hline & Sex & 0.95 & \\
\hline & Anxiety and depression & 1.02 & \\
\hline & Cognitive impairment & 1.24 & \\
\hline & Functional dependence & 1.05 & \\
\hline & Falls and fracture & 1.18 & \\
\hline & Incontinence & 1.04 & \\
\hline & Mobility problems & 1.09 & \\
\hline & Pressure ulcers & 1.04 & \\
\hline
\end{tabular}

dimensional prognostic index (MPI) AUC 0.617 HARP AUC 0.639 Charlson's co-morbidity index (CCI) AUC $0.579 ;^{44}>50$ years admitted to Intensive Care Unit (ICU) Clinical Frailty Scale (CFS) ORs for in-hospital mortality (1.81), adverse events(1.54), 1-year mortality(1.82), low quality of life score(1.98) and functional dependence $(2.25) ;{ }^{45}$ FI for patients admitted with hip fracture AUC 0.82 for failure to return home at 30 days; ${ }^{46}>65$ years admitted to hospital MPI AUC 0.76, FI-SOF AUC 0.68, FI-CD AUC 0.73, FI-CGA AUC 0.72 for all-cause mortality at 1 month; ${ }^{47}>80$ years admitted to hospital for at least $48 \mathrm{~h}$ via ED AUC 0.81 for functional decline at 2 months ${ }^{48}>70$ years admitted to acute geriatric ward CHS OR for mortality at 6 months CHS (4.68), SOF (1.97); >75 years admitted to acute care hospital, for every $1 \%$ increase in $F I$ is associated with a $5 \%$ increase in risk of death. ${ }^{49}$

We noted a phenomenon of improved predictive power reflected with in-spell models compared with historic models for both Charlson comorbidity scores and frailty syndromes. There may be two causes. First, HES data are coded at discharge, not admission. Diagnostic coding in HES may improve throughout the patients in-hospital stay with in-spell coding methodology adding an extra admission as a window for this to happen. Second, there may be 'leak' from the primary diagnostic coding position as these complex patients will likely have several reasons for emergency admission to hospital. Interestingly, taking into account comorbidity (by way of Charlson comorbidity score) did not significantly improve predictive power. VIF scores suggest only mild collinearity between the Charlson comorbidity score and frailty syndromes, suggesting mild overlap between the variables.

All our models displayed significance at $\mathrm{p}<0.05$ for the Hosmer-Lemeshow tests for goodness-of-fit test. Similar findings have been described by others who have produced models on HES specifically ${ }^{28}$ as the test is recognised to detect unimportant differences within large data sets. ${ }^{50}$ Ordering the patients by deciles of predicted risk allows a visual representation of the models discrimination.

\section{LIMITATIONS}

Though HES is a large data set with high information standards, it has limitations. It is retrospectively coded, thus reflects the patient's condition at discharge from hospital. To counter this, the model inputs data from 


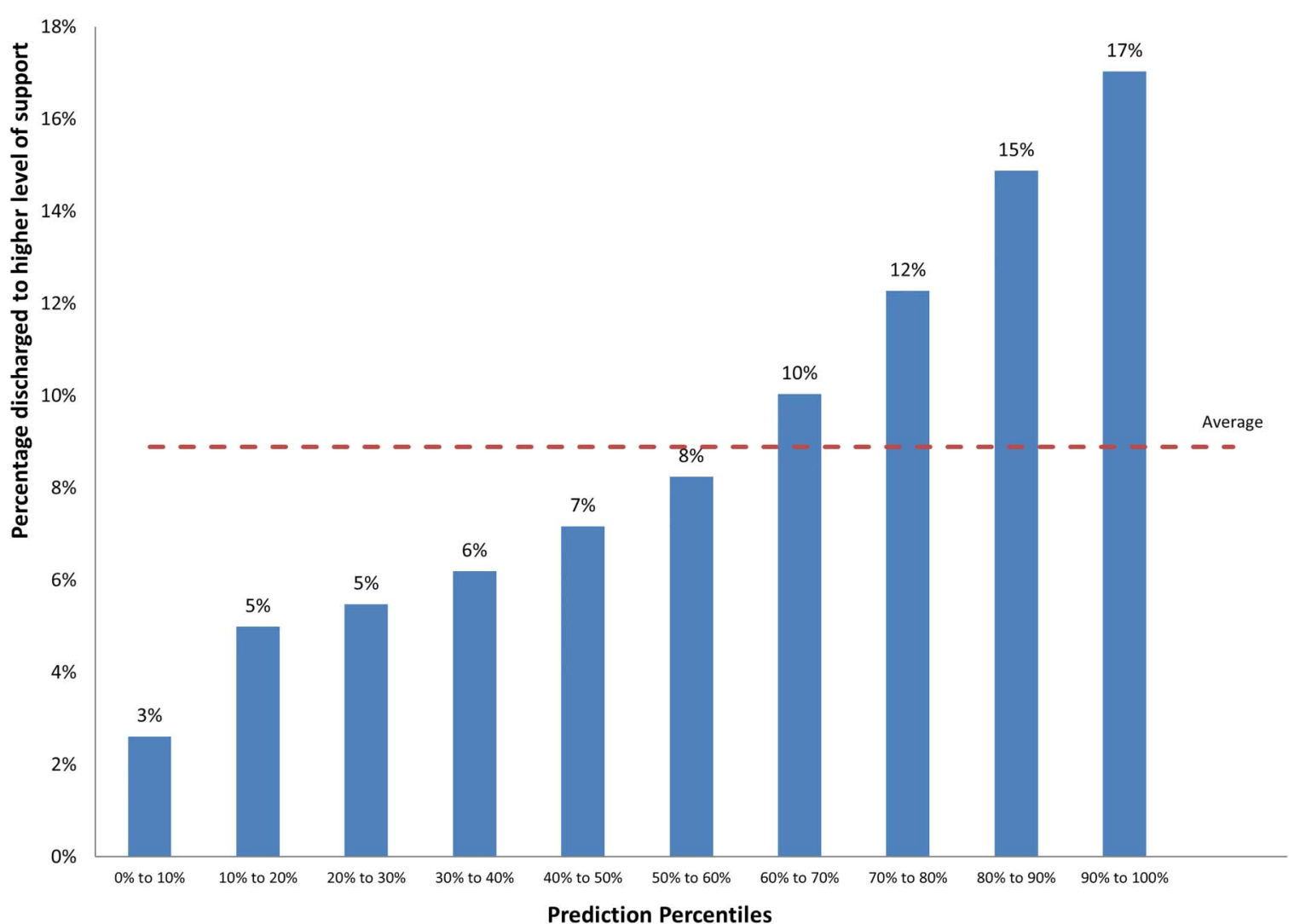

Figure 2 Percentage discharged to a higher level of functional dependence (institutionalisation) by prediction ranking for the frailty syndromes and admission source model.

historic spells to more accurately reflect a risk prediction tool at point of entry to care. Diagnostic coding accuracy in HES has been challenged. Plotting annual trend profiles of the data allowed us to choose a suitable temporal range to develop the model, as well as account for any change in coding practices over time. Even so, the administrative data set may not accurately reflect the actual clinical situation. Coding inconsistencies will limit

Table 7 Frailty syndrome models to predict emergency readmission within 30 days

\begin{tabular}{|c|c|c|c|}
\hline Model & ORs & & AUC \\
\hline \multirow[t]{9}{*}{ Historic frailty syndromes } & Age & 1.00 & 0.574 \\
\hline & Sex & 1.20 & \\
\hline & Anxiety and depression & 1.55 & \\
\hline & Cognitive impairment & 1.24 & \\
\hline & Functional dependence & 1.11 & \\
\hline & Falls and fracture & 1.25 & \\
\hline & Incontinence & 1.11 & \\
\hline & Mobility & 1.35 & \\
\hline & Pressure ulcers & 1.15 & \\
\hline \multirow[t]{11}{*}{ Historic frailty syndromes and admission history } & Age & 1.00 & 0.630 \\
\hline & Sex & 1.12 & \\
\hline & Anxiety and depression & 1.08 & \\
\hline & Cognitive impairment & 1.05 & \\
\hline & Functional dependence & 1.02 & \\
\hline & Falls and fracture & 1.03 & \\
\hline & Incontinence & 1.02 & \\
\hline & Mobility & 1.06 & \\
\hline & Pressure ulcers & 1.02 & \\
\hline & Number of emergency admissions (last $12 \mathrm{~m}$ ) & 1.47 & \\
\hline & Days since last emergency admission & 0.67 & \\
\hline
\end{tabular}

AUC, area under the curve. 


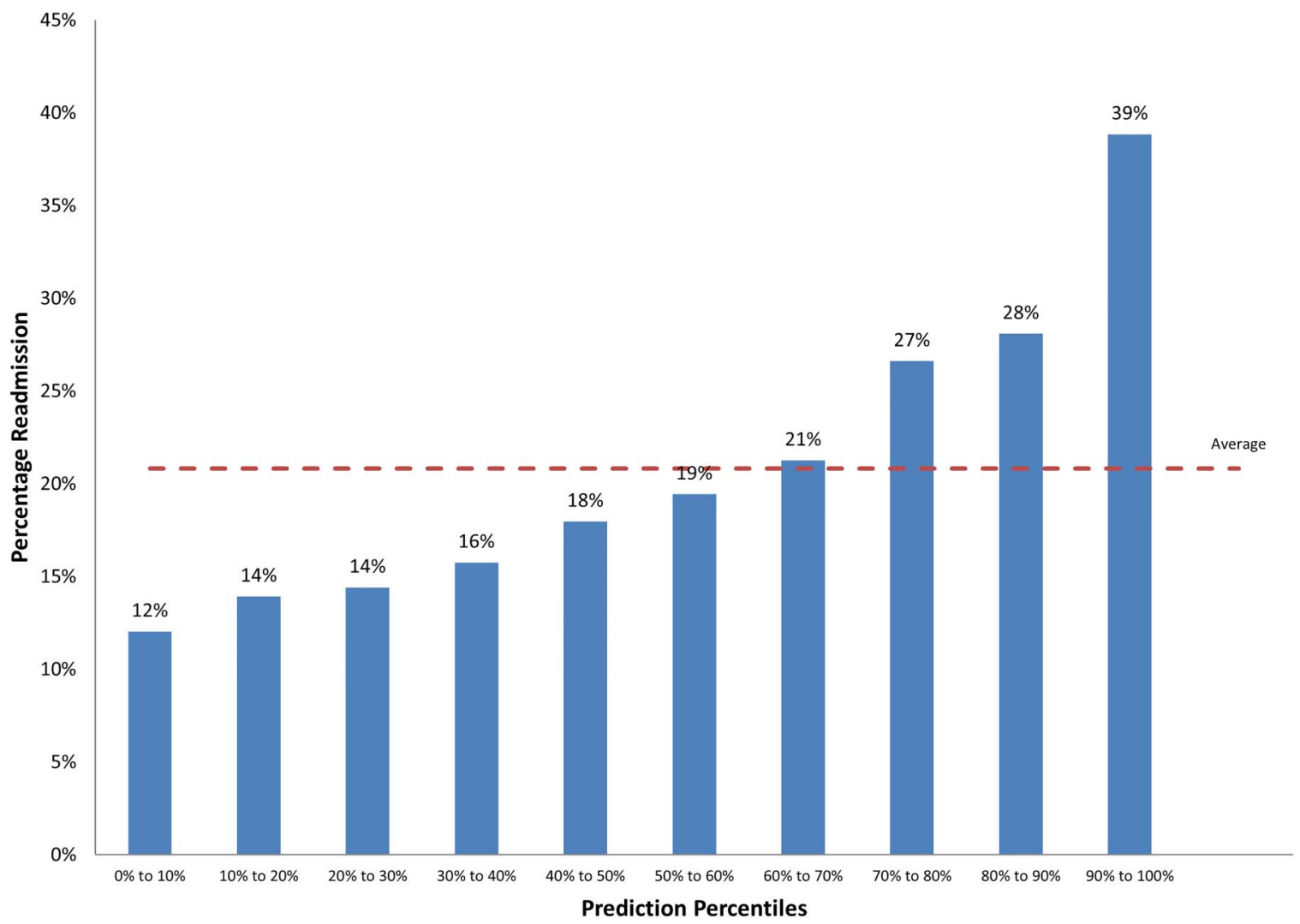

Figure 3 Percentage with emergency readmission within 30 days by prediction ranking for the frailty syndromes and admission history model.

the models predictive powers and accuracy. Prospective testing on a clinical data set is a necessary next step. Though a rich data set, HES does not contain variables previously identified as being predictive of frailty (eg, polypharmacy or weakness). This risks excluding potentially relevant variables from the model.
HES does not record specific clinical measures of functional dependency (eg, Barthel Index). The creation of a five-tier discharge institution levels represents a pragmatic approach to create an outcome that reflects increase in care need (within HES) as a proxy measure for increase in functional dependency. The premise of

Table 8 Summary of the predictive power of frailty scores in acute care

\begin{tabular}{|c|c|c|c|c|c|c|}
\hline \multirow{2}{*}{$\begin{array}{l}\text { Model/scores } \\
\text { AUCs }\end{array}$} & \multicolumn{2}{|l|}{ Mortality } & \multicolumn{2}{|c|}{ Readmission } & \multicolumn{2}{|c|}{ Functional dependence } \\
\hline & Inpatient & 90-day & 30-day & 90-day & Institutionalisation & $\leq 2$ points Barthel $A D L$ \\
\hline Charlson score 2012 (historic) & 0.64 & & 0.59 & & 0.62 & \\
\hline $\mathrm{CHS}$ model & & 0.61 & & 0.52 & 0.57 & 0.55 \\
\hline SOF model & & 0.59 & & 0.53 & 0.44 & 0.56 \\
\hline Avila-Funes & & 0.68 & & 0.55 & 0.50 & 0.59 \\
\hline Rothman & & 0.67 & & 0.53 & 0.45 & 0.59 \\
\hline Frailty Index & & 0.69 & & 0.57 & 0.55 & 0.57 \\
\hline ISAR & & 0.62 & & 0.60 & 0.65 & 0.60 \\
\hline PARR30 & & & 0.70 & & & \\
\hline RIGAMA & 0.78 & & 0.55 & & 0.50 & \\
\hline \multicolumn{7}{|l|}{ Frailty syndrome models } \\
\hline $\begin{array}{l}\text { Frailty syndromes and admission } \\
\text { source }\end{array}$ & & & & & 0.65 & \\
\hline Frailty syndromes & 0.62 & & 0.57 & & 0.63 & \\
\hline $\begin{array}{l}\text { Frailty syndromes and admission } \\
\text { history }\end{array}$ & 0.63 & & 0.63 & & 0.63 & \\
\hline
\end{tabular}


comparing discharge institution to admission source within HES as a surrogate for functional dependency is possibly flawed. Cohort and epidemiological studies suggest that there is significant overlap of functional dependency between residents of residential and nursing homes. Additionally, thresholds for transfer into and out of homes in the residential care setting are highly context and health system dependant. For instance, there is marked variation in the manner that criteria for NHS long-term funding are applied between geographical settings. However, the model adds new knowledge surrounding methodologies to utilise routinely collected data for answering clinically meaningful questions.

\section{CONCLUSION}

Frailty syndromes are a valid predictor of outcomes relevant to acute care. We provide a frailty score developed from routinely collected administrative data, and this study adds further understanding and utility for the secondary use of these data. The models predictive power is in keeping with other scores in the literature, but is a simple, clinically relevant and potentially more acceptable measurement for use in the acute care setting. Predictive powers of the score are not sufficient for clinical use, though HES coding quality in HES may be responsible. Prospective testing in a clinical data set and the addition of other variables known to predict frailty may improve predictive power. Frailty is an important dimension in risk stratification of older persons requiring acute care.

Contributors JS conceived study, designed analysis, interpreted results and wrote first draft. AJP designed analysis, interpreted results, contributed to ongoing writing. SS and KD designed analysis. DB conceived study, designed analysis, interpreted results and contributed to ongoing writing.

Funding This article presents independent research commissioned by the National Institute for Health Research (NIHR) under the Collaborations for Leadership in Applied Health Research and Care (CLAHRC) programme for North West London. JS received a Research Fellowship award from Chelsea and Westminster Health Charity. SS and KD seconded from Oliver Wyman to support this project.

\section{Competing interests None declared.}

Ethics approval As per Governance Arrangements for Research Ethics Committees (GAfREC), research limited to secondary use of information previously collected in the course of normal care (without an intention to use it for research at the time of collection), provided that the patients or service users are not identifiable to the research team in carrying out the research.

Provenance and peer review Not commissioned; externally peer reviewed.

Data sharing statement No additional data are available.

Open Access This is an Open Access article distributed in accordance with the terms of the Creative Commons Attribution (CC BY 4.0) license, which permits others to distribute, remix, adapt and build upon this work, for commercial use, provided the original work is properly cited. See: http:// creativecommons.org/licenses/by/4.0/

\section{REFERENCES}

1. Department for Environment, Food and Rural Affairs (Defra) Survey of public attitudes and behaviours towards the environment. 2011.
2. Marengoni A, Angleman S, Melis R, et al. Aging with multimorbidity: a systematic review of the literature. Ageing Res Rev 2011;10:430-9.

3. Wolff JL, Boult C, Boyd C, et al. Newly reported chronic conditions and onset of functional dependency. J Am Geriatr SoC 2005;53:851-5.

4. Department of Health. Improving care and saving money: learning the lessons on prevention and early intervention for older people. 2010.

5. Inouye SK, Studenski S, Tinetti ME, et al. Geriatric syndromes: clinical, research, and policy implications of a core geriatric concept. J Am Geriatr Soc 2007;55:780-91.

6. Heppenstall CP, Wilkinson TJ, Hanger HC, et al. Frailty: dominos or deliberation? N Z Med J 2009;122:42-53.

7. Ellis G, Whitehead MA, Robinson D, et al. Comprehensive geriatric assessment for older adults admitted to hospital: meta-analysis of randomised controlled trials. BMJ 2011;343:d6553.

8. Edmans J, Bradshaw L, Gladman JR, et al. The Identification of Seniors at Risk (ISAR) score to predict clinical outcomes and health service costs in older people discharged from UK acute medical units. Age Ageing 2013;42:747-53.

9. Wou F, Gladman JR, Bradshaw L, et al. The predictive properties of frailty-rating scales in the acute medical unit. Age Ageing 2013;42:776-81

10. Conroy S, Dowsing T. The ability of frailty to predict outcomes in older people attending an acute medical unit. Acute Med 2013;12 74-6.

11. Banerjee J, Conroy S, Cooke MW. Quality care for older people with urgent and emergency care needs in UK emergency departments. Emerg Med J 2013;30:699-700.

12. Acute Care Toolkit 3. Acute medical care for frail older people. London: Royal College of Physicians, 2012.

13. Edmans J, Bradshaw L, Franklin M, et al. Specialist geriatric medica assessment for patients discharged from hospital acute assessment units: randomised controlled trial. BMJ 2013;347:f5874

14. Rockwood K, Song X, MacKnight C, et al. A global clinical measure of fitness and frailty in elderly people. CMAJ 2005;173:489.

15. Rodriguez-Mañas L, Féart C, Mann G, et al. Searching for an operational definition of frailty: a Delphi method based consensus statement: the frailty operative definition-consensus conference project. J Gerontol A Biol Sci Med Sci 2013;68:62-7.

16. Morley JE, Vellas B, van Kan GA, et al. Frailty consensus: a call to action. J Am Med Dir Assoc 2013;14:392-7.

17. Fried LP, Tangen CM, Walston J, et al. Frailty in older adults: evidence for a phenotype. J Gerontol A Biol Sci Med Sci 2001;56: M146-56.

18. Wou F, Conroy S. The frailty syndrome. Medicine 2013;41:13-15.

19. Blodgett J, Theou O, Kirkland S, et al. Frailty in NHANES: comparing the frailty index and phenotype. Arch Gerontol Geriatr 2015;60:464-70.

20. Cesari M, Gambassi G, van Kan GA, et al. The frailty phenotype and the frailty index: different instruments for different purposes. Age Ageing 2014;43:10-12.

21. Isaacs $\mathrm{B}$. The challenge of geriatric medicine. USA: Oxford University Press, 1992.

22. Rockwood K, Mitnitski A, Song X, et al. Long-term risks of death and institutionalization of elderly people in relation to deficit accumulation at age 70. J Am Geriatr Soc 2006;54:975-9.

23. Anpalahan M, Gibson SJ. Geriatric syndromes as predictors of adverse outcomes of hospitalization. Intern Med J 2008;38:16-23.

24. HES Online: Hospital Episode Statistics. 2013. http://www.hscic. gov.uk/hes (accessed Nov 2014)

25. Rockwood K. What would make a definition of frailty successful? Age Ageing 2005;34:432-4.

26. Charlson ME, Pompei P, Ales KL, et al. A new method of classifying prognostic comorbidity in longitudinal studies: development and validation. J Chronic Dis 1987:40:373-83.

27. Health and social care information centre. Methodology to create provider and CIP spells from HES APC data: 1-9, 2014. http://www. hscic.gov.uk/media/11859/Provider-Spells-Methodology/pdf/Spells_ Methodology.pdf (accessed Nov 2014).

28. Bottle A, Aylin P. Comorbidity scores for administrative data benefited from adaptation to local coding and diagnostic practices. J Clin Epidemiol 2011;64:1426-33.

29. Pedregosa F, Varoquaux G, Granfort A, et al. Scikit-learn: machine learning in python. $J$ Mach Learn Res 2011;12:2825-30.

30. Lalkhen AG, McCluskey A. Clinical tests: sensitivity and specificity. Contin Educ Anaesth Crit Care Pain 2008;8:221-3.

31. Hosmer David W Jr, Lemeshow S, Sturdivant RX. Applied logistic regression (Wiley series in probability and statistics). Wiley-Blackwell, 2013:528. 2013-04-26. 
32. Wou F, Gladman JRF, Bradshaw L, et al. The predictive properties of frailty-rating scales in the acute medical unit. Age Ageing 2013;42:776-81.

33. Romero-Ortuno R, O'Dwyer C, Byrne D, et al. A Risk Index for Geriatric Acute Medical Admissions (RIGAMA). Acute Med 2014;13:6-11.

34. Billings J, Blunt I, Steventon A, et al. Development of a predictive model to identify inpatients at risk of re-admission within 30 days of discharge (PARR-30). BMJ Open 2012;2:e001667.

35. Kellett J, Clifford M, Ridley A, et al. A four item scale based on gait for the immediate global assessment of acutely ill medical patientsone look is more than 1000 words. Eur Geriatr Med 2014;5:92-6.

36. McCusker J, Bellavance F, Cardin S, et al. Detection of older people at increased risk of adverse health outcomes after an emergency visit: the ISAR screening tool. J Am Geriatr Soc 1999;47:1229-37.

37. Smith GB, Prytherch DR, Meredith $\mathrm{P}$, et al. The ability of the National Early Warning Score (NEWS) to discriminate patients at risk of early cardiac arrest, unanticipated intensive care unit admission, and death. Resuscitation 2013;84:465-70.

38. Yim VW, Rainer TH, Graham CA, et al. Emergency department intervention for high-risk elders: identification strategy and randomised controlled trial to reduce hospitalisation and institutionalisation. Hong Kong Med J 2011;17(3 Suppl 3):4-7.

39. Braes T, Flamaing J, Sterckx W, et al. Predicting the risk of functional decline in older patients admitted to the hospital: a comparison of three screening instruments. Age Ageing 2009;38:600-3.

40. Dendukuri N, McCusker J, Belzile E. The identification of seniors at risk screening tool: further evidence of concurrent and predictive validity. J Am Geriatr Soc 2004;52:290-6.

41. McCusker J, Bellavance F, Cardin S, et al. Prediction of hospital utilization among elderly patients during the 6 months after an emergency department visit. Ann Emerg Med 2000;36:438-45.
42. Cornette $\mathrm{P}$, Swine $\mathrm{C}$, Malhomme B, et al. Early evaluation of the risk of functional decline following hospitalization of older patients: development of a predictive tool. Eur J Public Health 2006;16: 203-8.

43. Sager MA, Rudberg MA, Jalaluddin M, et al. Hospital admission risk profile (HARP): identifying older patients at risk for functional decline following acute medical illness and hospitalization. J Am Geriatr Soc 1996;44:251-7.

44. Dent E, Chapman I, Piantadosi C, et al. Frailty determinants and discharge outcomes in hospitalised older persons. Australas $J$ Ageing 2012;31:71.

45. Bagshaw SM, Stelfox HT, McDermid RC, et al. Association between frailty and short- and long-term outcomes among critically ill patients: a multicentre prospective cohort study. CMAJ 2014;186: E95-102.

46. Krishnan M, Beck S, Havelock W, et al. Predicting outcome after hip fracture: using a frailty index to integrate comprehensive geriatric assessment results. Age Ageing 2014;43:122-6.

47. Pilotto A, Rengo F, Marchionni N, et al. Comparing the prognostic accuracy for all-cause mortality of frailty instruments: a multicentre 1-year follow-up in hospitalized older patients. PLOS ONE 2012;7: e29090.

48. Wu AW, Yasui Y, Alzola C, et al. Predicting functional status outcomes in hospitalized patients aged 80 years and older. $J$ Am Geriatr Soc 2000;48(5 Suppl):S6-15.

49. Evans SJ, Sayers M, Mitnitski A, et al. The risk of adverse outcomes in hospitalized older patients in relation to a frailty index based on a comprehensive geriatric assessment. Age Ageing 2014;43:127-32.

50. Hosmer DW, Hosmer T, Le Cessie S, et al. A comparison of goodness-of-fit tests for the logistic regression model. Stat Med 1997;16:965-80. 


\section{Correction}

Soong J, Poots AJ, Scott S, et al. Developing and validating a risk prediction model for acute care based on frailty syndromes. BMJ Open 2015;5:e008457. The corresponding author's email address is incorrect in this paper. The correct address is j.soong@ imperial.ac.uk

BMJ Open 2015;5:e008457corr1. doi:10.1136/bmjopen-2015-008457corr1

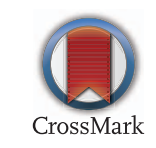

